\title{
ANÁLISES DE PROTOCOLOS DE BRAQUITERAPIA, POR ALTA TAXA DE DOSE, DO CONTROLE DE QUALIDADE DE ALGUNS SERVIÇOS LOCAIS, BASEADOS NO TG40, TG56 E ARCAL XXX*
}

\author{
Carmen S. Guzmán Calcina ${ }^{1,2}$, Adelaide de Almeida ${ }^{1}$, José R. Oliveira Rocha ${ }^{3}$
}

Resumo A braquiterapia por alta taxa de dose está recebendo atenção considerável na maioria dos países. Por isso, nos serviços que utilizam este equipamento exige-se que o desenvolvimento de um programa de controle de qualidade seja cada vez mais rigoroso, para garantir não apenas a segurança aos pacientes, mas também aos operadores e demais envolvidos. Este trabalho tem por objetivos fazer um levantamento dos tipos de testes para um equipamento de braquiterapia por alta taxa de dose, propostos pelos protocolos oficiais publicados (TG40, TG56 e ARCAL XXX) e avaliar os tipos de testes que atualmente são realizados por alguns serviços de radioterapia, comparando-os com aqueles apresentados nos protocolos citados. Das análises feitas, observou-se que: a) quanto aos protocolos oficiais, o TG56 é mais completo que o TG40 e o ARCAL XXX; b) quanto às instituições analisadas, estas em geral se basearam no TG56 para elaborar seus próprios protocolos, os quais demonstraram ter também concordância com os outros já citados. Nestes protocolos, a inexistência dos testes anuais foi notada, o que pode ser explicado por sua aparição nas frequiências trimestral e semestral. Do produto deste estudo são apresentadas tabelas dos tipos de testes com suas respectivas freqüências de utilização, das quais um protocolo pode ser inferido para auxiliar na implementação, pelo menos, dos tipos de testes de controle de qualidade básicos e indispensáveis para o equipamento, garantindo, assim, um tratamento adequado aos pacientes e uma melhor segurança ao pessoal envolvido e, conseqüentemente, assegurando a garantia de qualidade na braquiterapia por alta taxa de dose.

Unitermos: Dosimetria. Braquiterapia. Braquiterapia por alta taxa de dose (BATD). Controle de qualidade.

Abstract Analysis of the high dose rate brachytherapy protocols of quality assurance programs of some local services, based on TG40, TG56 and ARCAL XXX.

High dose rate brachytherapy has been increasingly recognized in most countries, and radiotherapy services using this equipment are encouraged to have a very efficient quality assurance program to ensure protection for patients, workers and other personnel involved. The objective of this paper was to determine the types of tests for high dose rate equipment required by official protocols (TG40, TG56 and ARCAL XXX) and to compare them with the types of tests utilized by some radiotherapy services. We concluded that: a) the protocol TG56 is more extensive and complete than the other official protocols (TG40 and ARCAL XXX); b) the protocols used by the services evaluated on this study were based on the protocol TG56, and were concordant with the other official protocols. In these protocols annual tests were frequently replaced by tests performed quarterly or twice a year. This study established the types of test used and their frequency of utilization, and permitted the design of an optimized protocol that may help in the implementation of basic and indispensable tests in order to ensure patient adequate treatment and safety to personnel involved, and consequently improve high dose rate brachytherapy quality assurance.

Key words: Dosimetry. Brachytherapy. High dose rate brachytherapy (HDR). Quality control.

\section{INTRODUÇÃO}

A braquiterapia é uma modalidade de tratamento que utiliza a radiação ionizante nos tumores malignos, e, para isso,

* Trabalho realizado no Departamento de Física e Matemática da Faculdade de Filosofia, Ciências e Letras de Ribeirão Preto (FFCLRP) da Universidade de São Paulo (USP), Ribeirão Preto, SP.

1. Departamento de Física e Matemática da FFCLRP-USP.

2. Departamento de Radioterapia, Instituto de Enferme dades Neoplásicas, Lima, Peru.

3. Setor de Física Médica do CEB-Unicamp e Setor de Radioterapia do CAISM-Unicamp, Campinas, SP.

Endereço para correspondência: Dra. Adelaide de Almeida. Departamento de Física e Matemática, FFCLRP-USP. Av. Bandeirantes, 3900. Ribeirão Preto, SP, 14040-901.

Aceito para publicação em 12/4/2001. faz uso de pequenas fontes radioativas seladas posicionadas a uma distância curta em relação ao volume alvo ${ }^{(\mathbf{1})}$. Estas fontes são radioisótopos cujas radiações, ao penetrarem o tecido de interesse, liberam toda ou parte de sua energia in loco, levando à morte as células neoplásicas ${ }^{(2)}$.

Em meados de 1980, a braquiterapia por alta taxa de dose ("high dose rate" HDR) foi desenvolvida como uma alternativa à braquiterapia por baixa taxa de dose ("low dose rate" - LDR). A braquiterapia com HDR, atualmente, está recebendo atenção considerável na maioria dos países ${ }^{(3)}$, em virtude de ser uma opção segura e eficiente na administração da dose de radiação no tratamento de tumor maligno em diferentes regiões do corpo $^{(1)}$. Por esse motivo, e por causa da complexidade da técnica de HDR, exige-se que os controles de segurança sejam cada vez mais rigorosos, com a finalidade de garantir a segurança, não somente aos pacientes, mas também aos operadores e demais envolvidos.

De acordo com a Organização Mundial da Saúde (OMS), o controle de qualidade (CQ) nos serviços de braquiterapia com HDR é fundamental, já que este controle deve garantir a consistência 
entre a dose clínica prescrita e a dose administrada ao paciente, levando em consideração o volume de interesse, as limitações da dose no tecido sadio, as exposições mínimas ao pessoal envolvido no trabalho e as verificações das dosimetrias e planejamento realizados ${ }^{(4,5)}$. O CQ permite a detecção e correção oportuna de erros que inviabilizariam um tratamento. Este é um processo contínuo, que deve ser constantemente avaliado para a sua própria otimização. Portanto, deve-se estar alerta às novas mudanças que serão consideradas nos protocolos de garantia de qualidade (GQ).

Diversos protocolos de CQ foram publicados desde o início da utilização da HDR, com a intenção de melhorar a GQ. Esses protocolos servem como guias para o desenvolvimento de um programa de CQ a ser implementado em serviços que fazem uso da HDR.

O trabalho proposto surgiu, inicialmente, da idéia de se avaliar quais eram os tipos de testes considerados importantes pelos programas de CQ de alguns protocolos oficiais internacionais mais utilizados, como o Task Group 40 (TG40) ${ }^{(6)}$, o Task Group 56 (TG56) ${ }^{(1)}$ e o protocolo ARCAL XXX ${ }^{(3)}$, além de publicações sobre o tema. No estudo também foram utilizados alguns protocolos de braquiterapia de alguns serviços de radioterapia locais nacionais e internacionais.

Considerando-se as diferenças entre todos os protocolos analisados, um mais abrangente foi obtido.

Um programa de GQ na braquiterapia por HDR consiste de verificações realizadas periodicamente. Para tal, é necessária a monitoração sistemática de medidas e de procedimentos, visando à qualidade e cuidados apropriados aos pacientes. Esse programa deve ser geral, incluindo os aspectos administrativos, clínicos, físicos e técnicos. Logo, um representante de cada categoria citada deverá fazer parte de uma equipe multidisciplinar que definirá critérios gerais ou padrões da qualidade definida que deverão ser seguidos pela instituição. Esta equipe está ligada ao desenvolvimento e/ou execução de tarefas específicas do programa de GQ, as quais são projetadas para minimizar as incertezas (randômicas ou sistemáticas) provenientes dos erros de instrumentação (falha mecânica ou elétrica) ou de operação (falta de atenção ou má-interpretação dos dados ou funcionamento do equipamento).

O CQ aplicado ao equipamento de HDR é composto por vários tipos de testes, que devem ser realizados periodicamente em todo o equipamento, avaliando seus desempenhos, os quais podem variar em conseqüência a defeitos eletrônicos e/ou falhas mecânicas. Uma disciplina rigorosa deverá nortear a freqüência da realização dos testes.

Como resultado deste trabalho, é apresentado um protocolo a ser aplicado no trabalho de rotina para os equipamentos de HDR, o qual enfatiza a periodicidade e níveis de tolerância para cada um dos tipos de testes.

\section{MATERIAIS E MÉTODOS}

O protocolo relatado pela American Association of Physicists in Medicine (AAPM), TG40, é considerado a contribuição mais importante a partir de 1994 e apresenta um programa geral de GQ em radioterapia, que atualiza e agrupa as recomendações mais importantes da publicação TG45 ${ }^{(7)}$.

O protocolo apresentado pela AAPM, TG56, descreve um "programa geral de GQ em radioterapia, especificamente para braquiterapia", que atualiza e agrupa as recomendações mais importantes das diferentes publicações, como o TG4 $1^{(8)}$ e o TG40 $0^{(6)}$.

O protocolo ARCAL XXX, publicado em 1999, é uma otimização do TG40, o qual proporciona, fundamentalmente, as recomendações para os aspectos físicos da GQ na braquiterapia, que serão normalmente implementadas por profissionais da área de física médica. Embora alguns aspectos clínicos também estejam incluídos nesse protocolo, esses são tratados de maneira superficial, somente para esclarecer certas pautas e atuações.

No presente trabalho também foram utilizados dois protocolos particulares nacionais e quatro internacionais, cujas instituições somente cederam seus dados com a garantia do sigilo quanto às suas identidades.
Foi feita uma intercomparação entre os protocolos oficiais internacionais, quanto aos tipos de testes existentes, e seu resultado foi utilizado numa intercomparação com aqueles protocolos das instituições locais nacionais e internacionais, levando-se em consideração os níveis de tolerância e frequiências dos tipos de testes analisados.

Os testes apresentados pelo TG40, quanto à periodicidade semanal, foram colocados juntamente com os testes diários apresentados pelos outros protocolos, já que os tipos de testes são os mesmos; também os testes apresentados pelo ARCAL XXX, quanto à periodicidade trimestral, foram considerados juntamente com os semestrais apresentados pelos outros protocolos, já que os tipos de testes são os mesmos.

\section{RESULTADOS E DISCUSSÃO}

Os resultados são mostrados em formas de tabelas, as quais poderiam, como sugestão, auxiliar no trabalho de rotina em braquiterapia.

Analisando-se os tipos de testes provenientes dos protocolos oficiais (Tabelas 1, 2 e 3), observou-se que, dos 131 tipos de testes apresentados, seis aparecem somente no TG40, 44 constam somente no TG56 e 17, somente no ARCAL XXX, sendo que sete são comuns ao TG40 e TG56, três ao TG40 e ARCAL XXX, um ao TG56 e ARCAL XXX, um é comum aos três protocolos citados e 52 tipos de testes são apresentados somente pelas instituições consideradas.

As Tabelas 1, 2 e 3 são provenientes de três protocolos oficiais, além de instituições nacionais $(\mathrm{N})$ e de quatro de instituições internacionais (I). Nessas tabelas, as três primeiras colunas apresentam o número dos tipos de testes dos protocolos oficiais, as outras colunas estão relacionadas às instituições estudadas.

A Tabela 4 apresenta os testes exclusivos a cada um dos protocolos oficiais e os comuns a ambos os protocolos, levando-se em conta também a periodicidade.

A Tabela 5 apresenta a intercomparação dos tipos de testes para a HDR, de todos os protocolos das instituições com relação aos oficiais. 
Tabela 1 Testes diários/semanais para o controle de qualidade do equipamento de alta taxa de dose.

\begin{tabular}{|c|c|c|c|c|c|c|c|}
\hline \multicolumn{3}{|c|}{$\begin{array}{l}\text { Protocolos } \\
\text { oficiais }\end{array}$} & \multicolumn{2}{|c|}{$\begin{array}{l}\text { Protocolos } \\
\text { locais }\end{array}$} & & \multirow{2}{*}{ Atividades } & \multirow{2}{*}{$\begin{array}{l}\text { Funcionamento, } \\
\text { inspeção, } \\
\text { verificação, } \\
\text { manutenção } \\
\text { e tolerância }\end{array}$} \\
\hline TG40 & TG56 & $\begin{array}{l}\text { ARCAL } \\
\text { XXX }\end{array}$ & $\mathrm{N}_{1}$ & $\mathrm{I}_{1}$ & & & \\
\hline & $\bullet$ & & & 1 & \multirow{8}{*}{$\begin{array}{l}\text { Função } \\
\text { do sistema } \\
\text { geral }\end{array}$} & Programação & Funcionando \\
\hline & $\bullet$ & & & 1 & & Ejeção da fonte & Funcionando \\
\hline & $\bullet$ & & & 1 & & Retração da fonte & Funcionando \\
\hline \multirow[t]{6}{*}{ - } & - & & & 4 & & $\begin{array}{l}\text { Indicador da luz durante tratamento: } \\
\text { Painel de controle }\end{array}$ & Verificado \\
\hline & $\bullet$ & & & 1 & & Interior do quarto de tratamento & Verificado \\
\hline & $\bullet$ & & & 1 & & Porta de ingresso & Verificado \\
\hline & $\bullet$ & & 1 & 1 & & Sistema de imagens & Funcionando \\
\hline & & & & 1 & & Suporte de tratamento & Verificado \\
\hline & & $\bullet$ & & & \multirow{3}{*}{$\begin{array}{l}\text { Sistema } \\
\text { de retorno } \\
\text { da fonte }\end{array}$} & Retorno manual & Disponível \\
\hline \multirow[t]{2}{*}{$\bullet$} & & $\bullet$ & & 1 & & Baterias de apoio & Funcionando \\
\hline & & & & 2 & & Compressor de ar & Funcionando \\
\hline$\bullet$ & & $\bullet$ & 1 & 4 & \multirow{5}{*}{$\begin{array}{l}\text { Guias } \\
\text { condutores } \\
\text { da fonte e } \\
\text { aplicadores }\end{array}$} & Integridade mecânica & Verificado \\
\hline$\bullet$ & & $\bullet$ & 1 & 4 & & Conectores limpos & Verificado \\
\hline \multirow[t]{8}{*}{ - } & & & & 3 & & Aplicadores, colpostato, cateteres, agulhas, etc. (antes do uso) & Inspecionar \\
\hline & & & & 1 & & Aplicadores, colpostato, cateteres, agulhas, etc. (depois do uso) & Manutenção feita \\
\hline & & & & 1 & & $\begin{array}{l}\text { Revisar possíveis danos após o uso de aplicadores, colpostato, } \\
\text { cateteres e agulhas }\end{array}$ & Inspecionado \\
\hline & • & & 1 & 4 & & $\begin{array}{l}\text { Data, tempo e atividade da fonte na unidade de tratamento e sistema } \\
\text { de planejamento }\end{array}$ & Verificado \\
\hline & $\bullet$ & & & 1 & $\begin{array}{l}\text { Precisão da } \\
\text { dose liberada }\end{array}$ & $\begin{array}{l}\text { Precisão da atividade da fonte e tempo comparado com um padrão } \\
\text { terciário }\end{array}$ & Verificado \\
\hline & $\bullet$ & & 2 & 3 & \multirow{12}{*}{$\begin{array}{l}\text { Segurança } \\
\text { ao paciente/ } \\
\text { público/equipe } \\
\text { de trabalho } \\
\text { (proteção } \\
\text { radiológica) }\end{array}$} & Monitor para checagem da radiação na área: som e/ou luz & Funcionando \\
\hline & & $\bullet$ & & 4 & & Monitor portátil de radiação & Funcionando \\
\hline & $\bullet$ & $\bullet$ & 2 & 4 & & $\begin{array}{l}\text { Sistema de comunicação áudio/visual, no painel ou no quarto da } \\
\text { enfermeira }\end{array}$ & Funcionando \\
\hline \multirow[t]{5}{*}{$\bullet$} & $\bullet$ & & & 2 & & Erro áudio/visual e indicadores de alarme & Verificado \\
\hline & $\bullet$ & & & 3 & & Instruções de emergência & Avaliado \\
\hline & $\bullet$ & & 1 & 3 & & Equipamentos de emergência & Avaliado \\
\hline & $\bullet$ & & & 3 & & Manual dos operadores & Avaliado \\
\hline & & & & 4 & & Interruptor caso o tempo de posicionamento da fonte seja excedido & Funcionando \\
\hline \multirow[t]{4}{*}{$\bullet$} & $\bullet$ & & 2 & 4 & & Interruptores de emergência: & \\
\hline & & • & 2 & 3 & & $\begin{array}{l}\text { porta } \\
\text { painel }\end{array}$ & $\begin{array}{l}\text { runcionando } \\
\text { Funcionando }\end{array}$ \\
\hline & & & 1 & 3 & & Interruptor na sala de tratamento & Funcionando \\
\hline & & & 1 & 3 & & Interruptor na sala do painel & Funcionando \\
\hline$\bullet$ & & & & & \multirow{7}{*}{$\begin{array}{l}\text { Posição } \\
\text { da fonte }\end{array}$} & Precisão da preparação da tira (auto-radiografia) & Verificado (1 mm) \\
\hline \multirow[t]{4}{*}{$\bullet$} & $\bullet$ & $\bullet$ & & 2 & & $\begin{array}{l}\text { Teste de precisão da posição primária para um cateter simples } \\
\text { (radiografia, "phantom") }\end{array}$ & Verificado \\
\hline & $\bullet$ & & & & & $\begin{array}{l}\text { Desvio da resposta da câmara de ionização localizada numa } \\
\text { posição programada }\end{array}$ & Verificado \\
\hline & $\bullet$ & & 1 & & & $\begin{array}{l}\text { Auto-radiografia de multicanais de cada posição usada durante } 0 \\
\text { tratamento e comparação com a posição programada esperada }\end{array}$ & Verificado \\
\hline & $\bullet$ & & & & & Posição relativa da ponta da fonte na régua & Verificado \\
\hline * & & & & & & Precisão da fonte e carregamento das fontes simuladoras & Verificado \\
\hline * & & & & 1 & & Reprodutibilidade da posição da fonte & Verificado \\
\hline
\end{tabular}


Tabela 1 Testes diários/semanais para o controle de qualidade do equipamento de alta taxa de dose. (continuação)

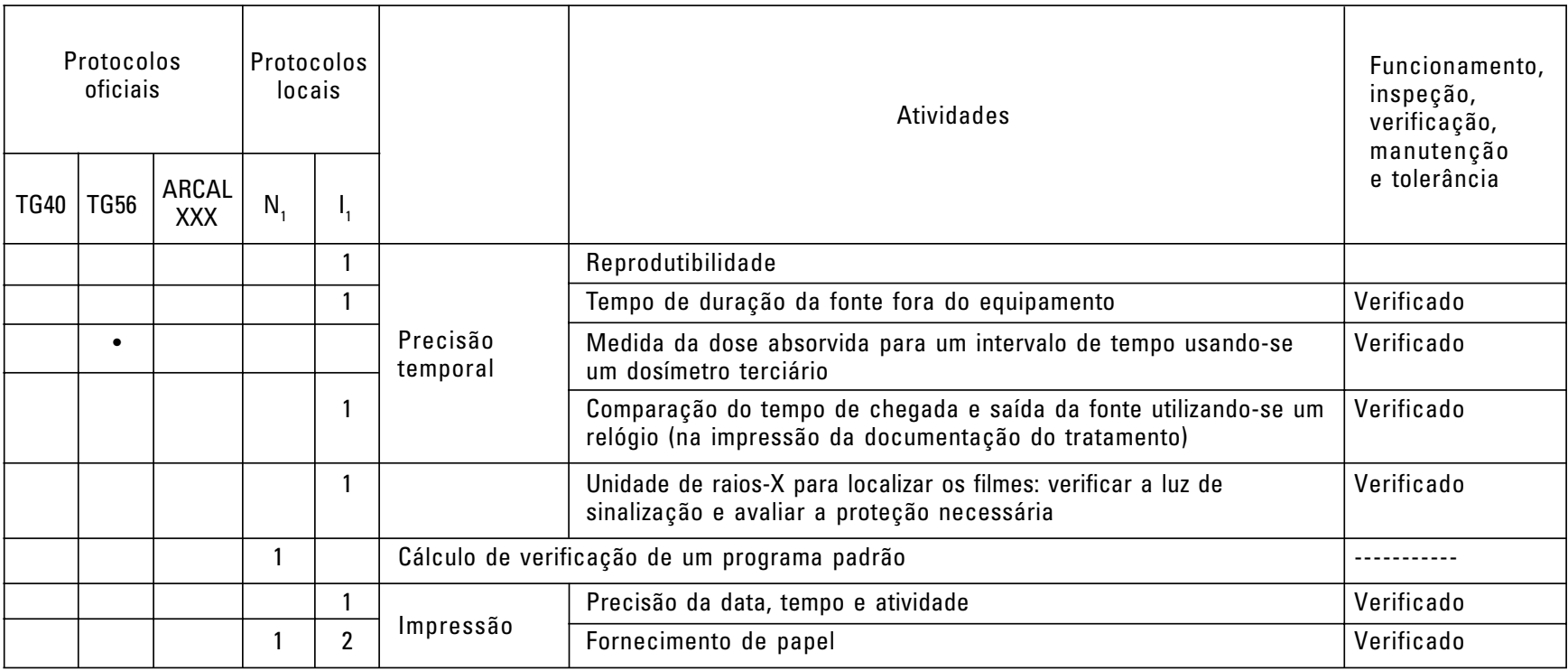

* São tipos de testes recomendados pelo TG40, semanalmente.

Tabela 2 Testes semestrais/trimestrais para o controle de qualidade do equipamento de alta taxa de dose.

\begin{tabular}{|c|c|c|c|c|c|c|c|}
\hline \multicolumn{3}{|c|}{$\begin{array}{l}\text { Protocolos } \\
\text { oficiais }\end{array}$} & \multicolumn{2}{|c|}{$\begin{array}{l}\text { Protocolos } \\
\text { locais }\end{array}$} & & \multirow{2}{*}{ Atividades } & \multirow{2}{*}{$\begin{array}{l}\text { Funcionamento, } \\
\text { inspeção, } \\
\text { verificação, } \\
\text { manutenção } \\
\text { e tolerância }\end{array}$} \\
\hline TG40 & TG56 & $\begin{array}{l}\text { ARCAL } \\
X X X\end{array}$ & $\mathrm{~N}_{1}$ & $\mathrm{I}_{1}$ & & & \\
\hline & $\bullet$ & * & & 1 & \multirow{10}{*}{$\begin{array}{l}\text { Segurança de pessoal } \\
\text { e paciente }\end{array}$} & Cabeça do equipamento com a fonte retraída & Inspecionado \\
\hline & & & 1 & 1 & & $\begin{array}{l}\text { Estabelecer os níveis de exposição do pessoal ocupacional } \\
\text { no ambiente de tratamento }\end{array}$ & -1----- \\
\hline & & & 1 & 1 & & Áreas adjacentes ao quarto de tratamento & Inspecionado \\
\hline & & & & 2 & & $\begin{array}{l}\text { Dosimetria pessoal essencialmente em situações que } \\
\text { requerem trocas de fontes }\end{array}$ & Verificado \\
\hline & & & 1 & & & Interruptor de emergência principal & Verificado \\
\hline & & & 1 & & & Interruptor de emergência auxiliar & Verificado \\
\hline & & & 1 & & & Interruptor de emergência da unidade de tratamento & Verificado \\
\hline & & & 1 & & & $\begin{array}{l}\text { Simular queda de tensão. Desligar o painel da unidade de } \\
\text { tratamento }\end{array}$ & --n-n-- \\
\hline & & \multirow[b]{2}{*}{ * } & & 1 & & \multirow{2}{*}{$\begin{array}{l}\text { Interruptores importantes e resposta de funcionamento de } \\
\text { sistemas de emergência: } \\
\qquad \begin{array}{c}\text { obstrução do aplicador } \\
\text { erro do aplicador }\end{array}\end{array}$} & Funcionando \\
\hline & & & & 1 & & & Funcionando \\
\hline & & & 1 & 1 & & Porta & Funcionando \\
\hline & & & & 1 & & Resolver a indexação & -.-.-.-.- \\
\hline & & & & 1 & & Deslocamento & Funcionando \\
\hline & & & & 1 & & Perda de pressão do ar & Verificado \\
\hline & & $*$ & & & & Revisão das fugas de ar & Verificado \\
\hline & & & 1 & & & Procedimento de teste das baterias & Verificado \\
\hline & $\bullet$ & & 1 & 1 & & Sistema de apoio de bateria & Verificado \\
\hline & & * & 1 & & & Perda de alimentação elétrica & Verificado \\
\hline & & $*$ & & & & Retorno das fontes ao "container" & Verificado \\
\hline
\end{tabular}


Tabela 2 Testes semestrais/trimestrais para o controle de qualidade do equipamento de alta taxa de dose. (continuação)

\begin{tabular}{|c|c|c|c|c|c|c|c|}
\hline \multicolumn{3}{|c|}{$\begin{array}{l}\text { Protocolos } \\
\text { oficiais }\end{array}$} & \multicolumn{2}{|c|}{$\begin{array}{l}\text { Protocolos } \\
\text { locais }\end{array}$} & & \multirow{2}{*}{ Atividades } & \multirow{2}{*}{$\begin{array}{l}\text { Funcionamento, } \\
\text { inspeção, } \\
\text { verificação, } \\
\text { manutenção } \\
\text { e tolerância }\end{array}$} \\
\hline \multirow[t]{5}{*}{ TG40 } & TG56 & $\begin{array}{l}\text { ARCAL } \\
\text { XXX }\end{array}$ & $\mathrm{N}_{1}$ & $\mathrm{I}_{1}$ & & & \\
\hline & & & & 1 & & $\begin{array}{l}\text { Integridade dos aplicadores, por dano mecânico, facilidade } \\
\text { de acoplamento, deformação mecânica nos enroscamentos }\end{array}$ & Verificado \\
\hline & - & & & & & $\begin{array}{l}\text { Ferramentas manuais de emergência para as fontes, } \\
\text { "container" com proteção, e avaliação para os aplicadores } \\
\text { de emergência para translado e funcionamento da } \\
\text { distribuição }\end{array}$ & Verificado \\
\hline & $\bullet$ & & & & $\begin{array}{l}\text { Calibração da posição } \\
\text { óptica e pneumática da } \\
\text { fonte/estado do sistema } \\
\text { de detecção da posição } \\
\text { ou estado da fonte, } \\
\text { qualquer outra inspeção } \\
\text { da manutenção } \\
\text { preventiva }\end{array}$ & Especificado pelo vendedor & $-\cdots-1-1$ \\
\hline & - & & 1 & 1 & \multirow{6}{*}{$\begin{array}{l}\text { Operação correta de } \\
\text { todo o aplicador, tubo } \\
\text { de transferência e } \\
\text { localização das "fontes } \\
\text { falsas" }\end{array}$} & $\begin{array}{l}\text { Exame de todas as "fontes falsas" por bloqueio que impede } \\
\text { seu deslocamento axial através do conjunto do aplicador }\end{array}$ & 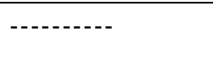 \\
\hline \multirow[t]{5}{*}{$\bullet$} & - & & & 1 & & Integridade da interface tubo-aplicador & Verificado \\
\hline & $\bullet$ & & & & & Trava do cabo de transferência & Verificado \\
\hline & • & & & 1 & & Aplicador não-inserido & Verificado \\
\hline & - & & & 1 & & Aplicador obstruído & Verificado \\
\hline & & & & 1 & & Simetria de proteção & Verificado \\
\hline \multirow[t]{8}{*}{$\bullet$} & & & & & \multirow{8}{*}{$\begin{array}{l}\text { Precisão da posição } \\
\text { da fonte }\end{array}$} & Precisão dos guias de fonte e conectores & Verificado $(1 \mathrm{~mm})$ \\
\hline & $\bullet$ & & 1 & 1 & & $\begin{array}{l}\text { Posição da fonte radioativa concorda com a marca da } \\
\text { "fonte falsa", testada contra uma boa posição usando } \\
\text { marcadores na simulação }\end{array}$ & $\begin{array}{l}\text { Verificado } \\
(0,5 \mathrm{~mm})\end{array}$ \\
\hline & - & & & & & Cabo de operação & Verificado \\
\hline & • & & 2 & 1 & & $\begin{array}{l}\text { Obtenção de auto-radiografias de multicanais com uma } \\
\text { seqüência em cada canal: verificar se o espaço da posição } \\
\text { está bom, designando uma seqüência boa para o canal } \\
\text { programado, e a longitude correta por indexação }\end{array}$ & $1 \mathrm{~mm}$ \\
\hline & - & & 1 & & & $\begin{array}{l}\text { Comprimento do tubo de transferência (se a estabilidade do } \\
\text { tempo não é confirmada e a precisão da posição é } \\
\text { influenciada pelo comprimento do tubo) }\end{array}$ & Verificado \\
\hline & \multirow{3}{*}{$\cdot$} & & & 1 & & Precisão de todas as marcas radiográficas & Verificado \\
\hline & & & 1 & & & Precisão do teste de posição diária & Verificado \\
\hline & & & & 1 & & $\begin{array}{l}\text { Posicionamento da proteção de tungstênio nos colpostatos } \\
\text { e aplicadores }\end{array}$ & $\begin{array}{l}\text { Documentado } \\
\text { Verificado }\end{array}$ \\
\hline \multirow[t]{5}{*}{$\cdot$} & - & & 1 & 1 & \multirow{3}{*}{ Calibração da fonte } & $\begin{array}{l}\text { Atividade de kerma no ar da fonte fazendo-se uso de um } \\
\text { padrão secundário }\end{array}$ & Inspecionado \\
\hline & $\bullet$ & * & & 2 & & $\begin{array}{l}\text { Diferença da atividade de kerma no ar entre a medida e a } \\
\text { especificada pelo vendedor dentro de uma margem } \\
\text { especificada }\end{array}$ & 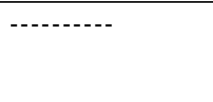 \\
\hline & $\bullet$ & & & & & $\begin{array}{l}\text { Uso da atividade de uma fonte terciária para confirmar a } \\
\text { calibração primária com um eletrômetro diferente e } \\
\text { detector a ser utilizado }\end{array}$ & $5 \%$ \\
\hline & • & & & 1 & & $\begin{array}{l}\text { Atividade da fonte sobre a data no registro do sistema de } \\
\text { planejamento, na unidade de tratamento e os inventários } \\
\text { semestrais }\end{array}$ & Verificado \\
\hline & $\bullet$ & * & & 2 & \multirow{2}{*}{ Temporizador } & Linearidade & - \\
\hline$\bullet$ & • & * & & 2 & & Reprodutibilidade & 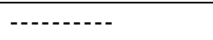 \\
\hline
\end{tabular}


Tabela 2 Testes semestrais/trimestrais para o controle de qualidade do equipamento de alta taxa de dose. (continuação)

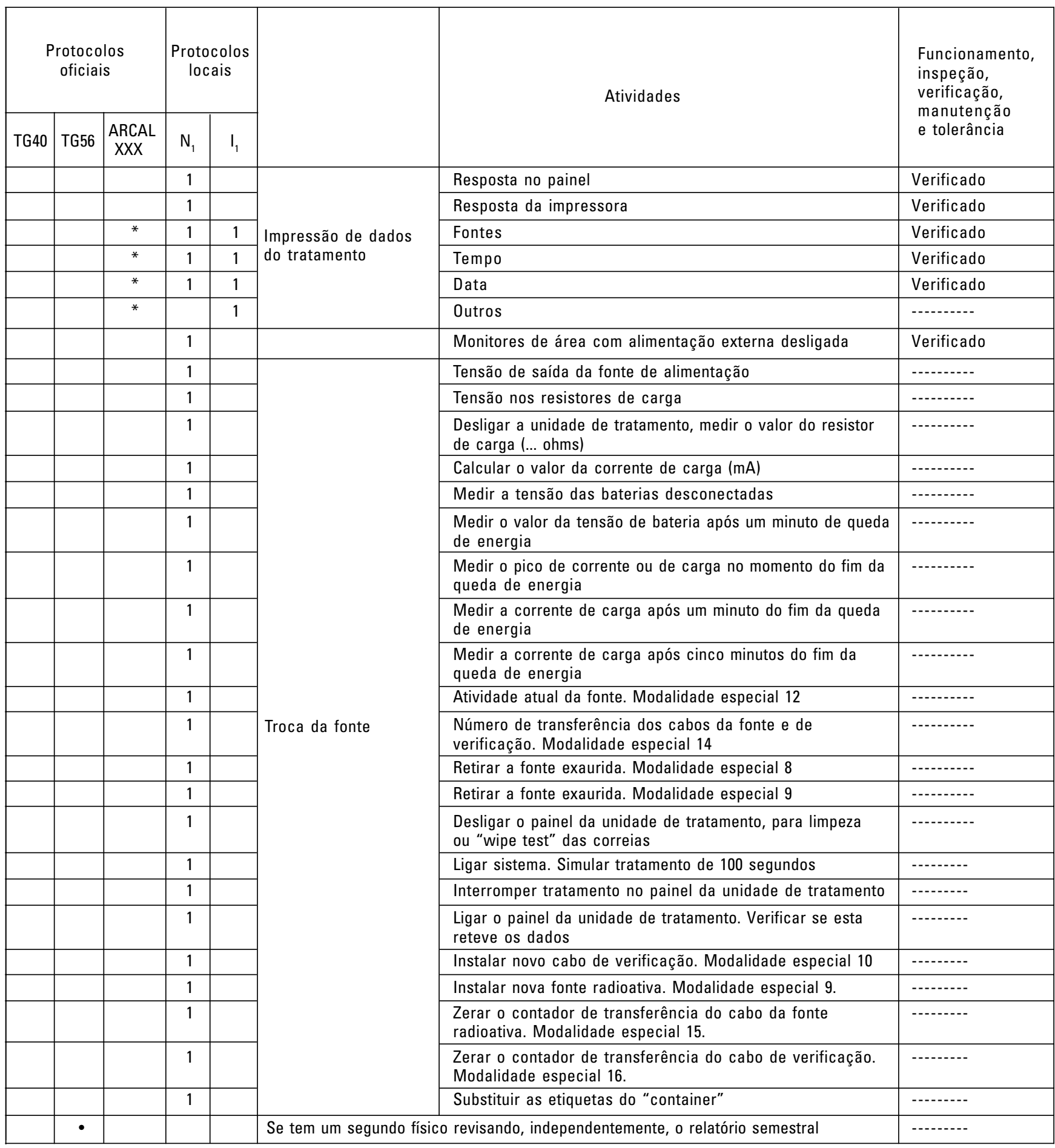

${ }^{*}$ ão tipos de testes recomendados pelo ARCAL XXX, trimestralmente.

Como resultado da fusão dos tipos de testes apresentados nos protocolos estudados, protocolos podem ser obtidos das Tabelas 1, 2 e 3, respectivamente para as freqüências diárias, semanais, semestrais, trimestrais e anuais, os quais poderiam auxiliar o trabalho de rotina na braquiterapia por HDR.

A princípio sugere-se que todos os testes apresentados nas tabelas, para as diferentes frequiências, sejam realizados pelas instituições. Entretanto, sabe-se que nem todas têm disponibilidade de todos os equipamentos e materiais necessários para suas realizações, mas, pelo menos, devem ser feitos aqueles testes básicos, os quais, em geral, apresentam freqüências maiores de uso. 
Tabela 3 Testes anuais para o controle de qualidade do equipamento de alta taxa de dose.

\begin{tabular}{|c|c|c|c|c|c|c|c|}
\hline \multicolumn{3}{|c|}{$\begin{array}{l}\text { Protocolos } \\
\text { oficiais }\end{array}$} & \multicolumn{2}{|c|}{$\begin{array}{l}\text { Protocolos } \\
\text { locais }\end{array}$} & & \multirow{2}{*}{ Atividades } & \multirow{2}{*}{$\begin{array}{l}\text { Funcionamento, } \\
\text { inspeção, } \\
\text { verificação, } \\
\text { manutenção } \\
\text { e tolerância }\end{array}$} \\
\hline TG40 & TG56 & $\begin{array}{l}\text { ARCAL } \\
X X X\end{array}$ & $\mathrm{~N}_{1}$ & $\mathrm{I}_{1}$ & & & \\
\hline & $\cdot$ & & & 1 & \multirow{3}{*}{$\begin{array}{l}\text { Segurança } \\
\text { do pessoal } \\
\text { e público }\end{array}$} & Carga de trabalho na área não-restrita à exposição & Revisado \\
\hline \multirow[t]{4}{*}{$\bullet$} & • & & & 1 & & Estrutura na construção & Inspecionar \\
\hline & & • & & & & Testes de fugas de radiação & \\
\hline & & • & & 1 & \multirow{2}{*}{$\begin{array}{l}\text { Precisão } \\
\text { de dose } \\
\text { liberada }\end{array}$} & Atividade da fonte & Verificado \\
\hline & $\bullet$ & & & 1 & & $\begin{array}{l}\text { Intercomparação com um padrão secundário usado para a } \\
\text { calibração semestral contra outro padrão departamental }\end{array}$ & $\mid--\cdot-\cdot--\cdot$ \\
\hline & $\bullet$ & & & & \multirow{3}{*}{$\begin{array}{l}\text { Precisão } \\
\text { da posição } \\
\text { da fonte }\end{array}$} & $\begin{array}{l}\text { Precisão de cassetes auto-radiográficos usados para as verificações } \\
\text { da precisão da posição }\end{array}$ & Verificado \\
\hline & $\bullet$ & & & & & Construção/espaçamento de toda marca simuladora ("fontes falsas") & Verificado \\
\hline & $\bullet$ & & & & & $\begin{array}{l}\text { Posição das marcas simuladoras para todo tipo de aplicador. } \\
\text { Verificação do procedimento da localização da fonte simuladora }\end{array}$ & Verificado \\
\hline & $\bullet$ & & & & \multirow{2}{*}{$\begin{array}{l}\text { Precisão } \\
\text { temporal }\end{array}$} & Linearidade do tempo e da precisão absoluta & Verificado \\
\hline & $\bullet$ & & & & & Dose de trânsito e da velocidade da fonte & Verificado \\
\hline & $\bullet$ & & & & \multirow{4}{*}{ Emergência } & $\begin{array}{l}\text { Unidades detectadas de simulação, fontes capsuladas estão } \\
\text { deslocadas }\end{array}$ & Verificado \\
\hline & • & & & & & Interruptores de emergência na sala e retração manual da fonte & Verificado \\
\hline \multirow[t]{2}{*}{$\bullet$} & & & & 1 & & $\begin{array}{l}\text { Simular um procedimento de emergência que poderia acontecer } \\
\text { quando o pessoal está em operação e avaliar as decisões a serem } \\
\text { tomadas }\end{array}$ & $-\cdots-\cdot--\cdot$ \\
\hline & $\bullet$ & & & & & $\begin{array}{l}\text { Motor de emergência de retração da fonte ativado quando há } \\
\text { excessiva fricção na obstrução do aplicador, durante deslocamento } \\
\text { da fonte }\end{array}$ & Verificado \\
\hline
\end{tabular}

Das análises feitas dos protocolos internacionais utilizados como padrões, os resultados dos testes diários, semestrais e anuais estão apresentados na Tabela 4.

Quanto às instituições estudadas e à periodicidade dos testes, os resultados são mostrados na Tabela 5 .

\section{CONCLUSÕES}

\section{Quanto aos protocolos oficiais}

Das análises feitas dos protocolos oficiais, observou-se que o protocolo TG56 é mais completo que o TG40 e o ARCAL XXX. Percebe-se que, neste último, há uma preocupação maior com os testes referentes à teleterapia.

\section{Quanto às instituições}

As instituições analisadas se basearam, em geral, no TG56 para elaborar seus próprios protocolos. Estes últimos também demonstraram ter uma concordância com os outros já citados.

Tabela 4 Distribuição dos testes nos protocolos internacionais utilizados, ou nas suas combinações para freqüências diárias, semanais, semestrais, trimestrais e anuais.

\begin{tabular}{|l|c|c|c|c|c|}
\hline \multicolumn{1}{|c|}{ Protocolos } & Diário & Semanal & Semestral & Trimestral & Anual \\
\hline TG40 & 2 & 2 & 1 & 0 & 1 \\
TG56 & 16 & 0 & 18 & 0 & 10 \\
ARCAL & 3 & 0 & 0 & 12 & 2 \\
TG40-TG56 & 3 & 0 & 3 & 0 & 1 \\
TG40-ARCAL & 3 & 0 & 0 & 0 & 0 \\
TG56-ARCAL & 1 & 0 & 0 & 0 & 0 \\
TG40-TG56-ARCAL & 1 & 0 & 0 & 0 & 14 \\
Total de testes & 29 & 2 & 22 & 12 & 14 \\
\hline
\end{tabular}

Das análises dos protocolos particulares, notou-se a inexistência quanto aos tipos de testes anuais, o que pode ser explicado pelo aparecimento destes nas freqüências trimestral e semestral.

De modo geral, as instituições estudadas desenvolveram um CQ de acordo com a sua disponibilidade de instrumentação, de pessoal e de tempo.

\section{Quanto ao controle de qualidade}

Para se implementar um programa adequado de GQ, os recursos financeiros e uma administração eficiente são imprescindíveis para se ter os equipamentos e materiais necessários, além de um grupo adequadamente capacitado.

É necessário que todos os serviços de braquiterapia implementem os tipos de 
Tabela 5 Distribuição de número dos tipos de testes nas instituições locais (nacionais e internacionais) em relação aos protocolos estudados para freqüências: a) diárias-semanais; b) semestrais-trimestrais; c) anuais.

\begin{tabular}{|l|c|c|c|c|c|c|}
\hline a) Diária/semanal & $\mathrm{N}_{1}$ & $\mathrm{~N}_{2}$ & $\mathrm{I}_{1}$ & $\mathrm{I}_{2}$ & $\mathrm{I}_{3}$ & $\mathrm{I}_{4}$ \\
\hline TG40 & 0 & 0 & 1 & 2 & 1 & $1 / 1$ \\
TG56 & 5 & 2 & 10 & 4 & 2 & 6 \\
ARCAL & 1 & 1 & 2 & 4 & 2 & 2 \\
TG40-TG56 & 1 & 1 & 2 & 1 & 3 & 0 \\
TG40-ARCAL & 2 & 0 & 2 & 3 & 2 & 2 \\
TG56-ARCAL & 1 & 1 & 1 & 1 & 1 & 1 \\
TG40-TG56-ARCAL & 0 & 0 & 1 & 0 & 0 & 1 \\
Instituição local & 2 & 2 & 4 & 6 & 7 & 7 \\
Total de testes & 12 & 7 & 23 & 21 & 18 & 21 \\
\hline b) Semestral/trimestral & $\mathrm{N}_{1}$ & $\mathrm{~N}_{2}$ & $\mathrm{I}_{1}$ & $\mathrm{I}_{2}$ & $\mathrm{I}_{3}$ & $\mathrm{I}_{4}$ \\
\hline TG40 & 0 & 0 & 0 & 0 & 0 & 0 \\
TG56 & 6 & 1 & 0 & 0 & 2 & 7 \\
ARCAL & 3 & 0 & 0 & 4 & 0 & 1 \\
TG40-TG56 & 1 & 0 & 0 & 0 & 1 & 2 \\
TG40-ARCAL & 0 & 0 & 0 & 0 & 0 & 0 \\
TG56-ARCAL & 0 & 0 & 0 & 0 & 0 & 2 \\
TG40-TG56-ARCAL & 0 & 0 & 0 & 0 & 0 & 0 \\
Instituição local & 34 & 0 & 0 & 2 & 1 & 10 \\
Total de testes & 44 & 1 & 0 & 6 & 4 & 22 \\
\hline
\end{tabular}

Obs.: Embora as instituições façam alguns testes semestralmente, estes coincidem com aqueles apresentados para o ARCAL trimestralmente

\begin{tabular}{|l|c|c|c|c|c|c|}
\hline C) Anual & $\mathrm{N}_{1}$ & $\mathrm{~N}_{2}$ & $\mathrm{I}_{1}$ & $\mathrm{I}_{2}$ & $\mathrm{I}_{3}$ & $\mathrm{I}_{4}$ \\
\hline TG40 & 0 & 0 & 0 & 1 & 0 & 0 \\
TG56 & 0 & 0 & 0 & 1 & 1 & 0 \\
ARCAL & 0 & 0 & 0 & 0 & 1 & 0 \\
TG40-TG56 & 0 & 0 & 0 & 1 & 0 & 0 \\
TG40-ARCAL & 0 & 0 & 0 & 0 & 0 & 0 \\
TG56-ARCAL & 0 & 0 & 0 & 0 & 0 & 0 \\
TG40-TG56-ARCAL & 0 & 0 & 0 & 0 & 0 & 0 \\
Instituição local & 0 & 0 & 0 & 0 & 0 & 0 \\
Total de testes & 0 & 0 & 0 & 3 & 2 & 0 \\
\hline
\end{tabular}

testes de CQ básicos e indispensáveis para seu equipamento, garantindo, desse modo, um tratamento adequado a seus pacientes e segurança ao pessoal técnico envolvido.

\section{Agradecimentos}

À Capes, pelo suporte financeiro, e às instituições que cederam suas informações.

\section{REFERÊNCIAS}

1. Nath R, Anderson LL, Meli JA, Olch AJ, Stitt JA, Williamson JF. Code of practice for brachytherapy physics: report of the AAPM Radiation Therapy Committee Task Group No. 56. American Association of Physicysts in Medicine. Med Phys 1997; 24:1557-98.

2. Fletcher GH. Textbook of radiotherapy. Philadelphia: Lea \& Febiger, 1974

3. Organismo Internacional de Energía Atómica dentro de las Actividades del Programa Regional ARCAL XXX. Aspectos físicos de la garantía de calidad en radioterapia, 1999.

4. World Health Organization. Quality assurance in radiotherapy. Génève: WHO, 1988.

5. Starkschall G, Horton J. Quality assurance in radiotherapy physics. Madison, Wisc.: 1991.

6. Kutcher GJ, Coia L, Gillin M, et al. Comprehensive QA for radiation oncology: report of AAPM Radiation Therapy Committee Task Group 40. Med Phys 1994;21:581-618.

7. Kubo HD, Glasgow GP, Pethel TD, Thomadsen BR, Williamson JF. High dose rate brachytherapy treatment delivery: report of the AAPM Radiation Therapy Committee Task Group No. 59. Med Phys 1998;25:375-403.

8. Glasgow GP, Bourland JD, Grigsby PW, Mely JA, Weaver KA. Remote afterloading technology. Report of Task Group 41. New York: American Institute of Physics, 1993.

9. International Commission on Radiation Units and Measurements. Determination of absorbed dose in a patient irradiated by beams of $\mathrm{X}$ or gamma rays in radiotherapy procedures. Washington, USA: ICRU 24, 1976. 